Revisión bibliográfica

Volumen 32(3): 1000-1018. Septiembre-diciembre, 2021 e-ISSN 2215-3608, doi:10.15517/am.v32i3.40607 https://revistas.ucr.ac.cr/index.php/agromeso/index

\title{
Calidad composicional y sensorial de la carne bovina y su determinación mediante infrarrojo cercano ${ }^{1}$
}

\section{Compositional and sensory quality of beef and its determination by near infrared}

\author{
Wilson Andrés Barragán-Hernández ${ }^{2,3,4}$, Liliana Mahecha-Ledesma ${ }^{3}$, Martha Olivera-Angel ${ }^{4}$, \\ Joaquín Angulo-Arizala ${ }^{3}$
}

1 Recepción: 10 de agosto, 2020. Aceptación: 1 de febrero, 2021. El trabajo formó parte de la tesis de Doctorado en Ciencias Animales del primer autor "Determinación de la calidad de carne bovina y la aceptación por parte del consumidor mediante el uso de pruebas con base en infrarrojo cercano". Universidad de Antioquia, Facultad de Ciencias Agrarias. Medellín, Colombia.

2 Corporación Colombiana de Investigación Agropecuaria (AGROSAVIA), Centro de Investigación El Nus. San Roque, Colombia. wbarraganh@agrosavia.co (https://orcid.org/0000-0003-3528-4296).

3 Universidad de Antioquia, Facultad de Ciencias Agrarias, Grupo de Investigación en Ciencias Agrarias (GRICA). AA1126, Ciudadela Robledo, Calle 70 No 52-21. Medellín, Colombia. Teléfono +57 (4) 2199147. liliana.mahecha@udea.edu.co (autora para la correspondencia; http://orcid.org/0000-0003-3377-8399); joaquin.angulo@udea.edu (https://orcid.org/0000-0003-3352-8795).

4 Universidad de Antioquia, Facultad de Ciencias Agrarias, Grupo de Investigación Biogénesis. AA1126, Ciudadela Robledo, Calle 70 No 52-21. Medellín, Colombia. martha.olivera@udea.edu.co (https://orcid.org/0000-0001-7853-4406).

\section{Resumen}

Introducción. La carne bovina es un alimento de alto valor biológico para la nutrición humana, es fuente de grasa, proteína, vitaminas y minerales. Sin embargo, aspectos asociados al impacto ambiental del sistema productivo y asociación con enfermedades no trasmisibles, han promovido nuevas tendencias en el consumo de proteína (vegetal, insectos, laboratorio), por lo que se hace necesario identificar y ofrecer información sobre los atributos de la carne que favorecen la aceptación de los consumidores, de forma rápida y confiable a través de técnicas no destructivas y amigables al ambiente como el infrarrojo cercano. Objetivo. Revisar las experiencias asociadas a la implementación de la Espectroscopia de Infrarrojo Cercano (NIRS) como alternativa en la determinación de la calidad de la carne bovina. Desarrollo. Los atributos de la carne con mayor relación con los consumidores pueden ser segmentados en apariencia, consumo y confianza. Estas características involucran elementos intrínsecos de la carne como color, grasa y terneza. Dada las limitantes en la aplicación de técnicas de laboratorio para la determinación de atributos cárnicos, se revisa la aplicación del NIRS con experiencias exitosas en características de interés para el consumidor. Además, se estudia la aplicación del NIRS en líneas de producción y otros usuarios de interés en la industria como detección de anomalías en la carne y autenticación de producto. Conclusión. La aplicación del NIRS, permite determinar de forma rápida y confiable atributos de la calidad de la carne que tienen una estrecha relación con el consumidor. Esta herramienta permite obtener resultados que respondan a la velocidad de producción de la industria y discriminar y autenticar aspectos relacionados con la carne y el sistema de producción.

Palabras claves: espectroscopia infrarroja, carne de res, consumidor, percepción. 


\begin{abstract}
Introduction. Beef is a food of high biological value for human nutrition; it is a source of fat, protein, vitamins, and minerals. However, aspects associated with the environmental impact of the production system and association with non-communicable diseases have promoted new protein consumption trends (vegetable, insects, lab protein). So, it is necessary to identify and offer information on the attributes of the meat that favor consumer acceptance quickly and reliably through non-destructive and environmentally friendly techniques such as near-infrared. Objective. To review the experiences associated with the implementation of Near-Infrared Spectroscopy (NIRS) as an alternative in the determination of beef quality. Development. Meat attributes with the greatest relationship with consumers can be segmented into appearance, consumption, and confidence. These characteristics involve intrinsic elements of the meat, such as color, fat, and tenderness. Given the limitations in the application of laboratory techniques for the determination of meat attributes, the application of the NIRS is reviewed with successful experiences in characteristics of interest to consumers. Additionally, the application of NIRS in production lines and other applications of interest in the industry, such as detection of meat anomalies and product authentication, is studied. Conclusion. The application of the NIRS allows to determine quickly and reliably attributes of the quality of the meat that have a close relationship with the consumer. This tool allows to obtain results that respond to the production speed of the industry, and to determinate and authenticate aspects related to meat and the production system.
\end{abstract}

Keywords: infrared spectrophotometry, meat, consumers, perception.

\title{
Introducción
}

La carne bovina, en el contexto de una dieta balanceada, se considera un alimento de alto valor biológico para la nutrición humana, es fuente de proteína, grasa, vitaminas y minerales (Scollan et al., 2017). Se ha reportado que un consumo de carne por adultos en Europa puede variar entre 75 y 211 g por día, con lo cual se cubren entre 12 $\%$ y $17 \%$ de los requerimientos diarios de energía, de $29 \%$ a $41 \%$ de proteína, de $19 \%$ a $24 \%$ de grasa, entre $4 \%$ y $38 \%$ de vitamina B, de $5 \%$ a $50 \%$ de vitamina D y de $16 \%$ y $13 \%, 27 \%$ a $23 \%, 19 \%$, y $36 \%$ de los requerimientos de minerales en hierro, potasio y zinc, respectivamente (Cocking et al., 2020). Sin embargo, la importancia nutricional de la carne no es consecuente con la percepción de los consumidores, la cual involucra una serie de factores sobre la carne que se relacionan con su preferencia y elección de compra (Barragán-Hernández et al., 2021b; Font-i-Furnols \& Guerrero, 2014; Henchion et al., 2017).

La industria de la carne bovina se ha visto afectada por el impacto ambiental sobre los sistemas de producción y por la asociación del consumo con la presencia de enfermedades no trasmisibles (Ekmekcioglu et al., 2018; Sakadevan \& Nguyen, 2017). Esta situación ha favorecido posiciones en pro de la reducción parcial o total de las carnes rojas en la dieta (Willett et al., 2019) y la tendencia en el consumo de fuentes proteicas en favor de sustitutos vegetales, homólogos de carne con base en insectos y carne producida en laboratorio (Joseph et al., 2020).

A pesar de las tendencias crecientes en alternativas proteicas de la carne, los consumidores demuestran preferencia por la carne bovina, fundamentadas en su sabor, textura y familiaridad del producto (Miller, 2020). Se ha demostrado que, los consumidores prefieren carne de bovino de granja (72\%), versus sustitutos con base en vegetales (21\%) y carne de laboratorio (5\%) (Van Loo et al., 2020). Esta situación, abre una ventana para explotar aquellos atributos de la carne que favorecen la aceptación del consumidor, centradas en factores intrínsecos y extrínsecos de la carne mediante la entrega de información precisa y confiable a los consumidores. Al respecto, se ha demostrado que la presentación de marcas o sellos (Meyerding et al., 2018) y el aportar información nutricional (Flowers, et al., 2018a; Yang et al., 2020), favorecen la aceptación y preferencia de la carne por parte de los consumidores. 
Los atributos intrínsecos y extrínsecos de la carne han sido bien documentados en la literatura (Grunert et al., 2004; Henchion et al., 2017), al igual que la interacción de los consumidores con los atributos, tales como preferencia y percepción (McCarthy et al., 2017). Por ejemplo, entre los atributos intrínsecos de mayor interés por parte de los consumidores se citan el color, la grasa intramuscular, la terneza y la percepción general del producto (Aboah \& Lees, 2020), los cuales pueden tener calificaciones positivas o negativas, dependiendo del perfil del consumidor, sexo, nivel educativo, frecuencia de consumo, entre otros (Ardeshiri \& Rose, 2018; Arenas-DeMoreno et al., 2020; Ngapo et al., 2017).

Desde el punto de vista objetivo, atributos de la carne de alto impacto en la percepción del consumidor pueden ser medidos en laboratorio bajo procedimientos que involucran el uso de equipos y reactivos, con tiempos que pueden ir desde pocos segundos (evaluación del color con espectrofotómetro) a algunas horas (determinación de grasa con método soxhlet), como puede ser revisado en Font-i-Furnols et al. (2015). Esta situación representa la necesidad de depender de varios equipos para la identificación de los atributos de la calidad de la carne y la posibilidad de emitir residuos contaminantes derivados de los reactivos utilizados en el proceso de análisis.

La aplicación de la Espectroscopia de Infrarrojo Cercano (NIRS), se ha planteado como una alternativa de análisis de los atributos de la calidad de la carne de forma rápida, confiable y no destructiva (Prieto et al., 2017). La técnica se basa en la interacción de la luz con moléculas orgánicas (C-H, N-H, H-O, entre otras) en el rango del espectro electromagnético que puede incluir tanto la región visible (VIS, 400 a $800 \mathrm{~nm}$ ), como la región cercana al infrarrojo (NIR, 800 - $2400 \mathrm{~nm}$ ) y la aplicación de modelos quimiométricos para el procesamiento de datos (Workman, 2016).

La situación actual de la industria cárnica y el creciente requerimiento de los consumidores por conocer las características de la carne que consume (Aboah \& Lees, 2020) y sus contenidos nutricionales (Flowers et al., 2019), demandan de métodos de cuantificación rápidos de los atributos de la calidad, a fin de responder con la velocidad y exigencias que demanda la cadena de producción. Por tal motivo, el presente documento plantea como objetivo revisar las experiencias asociadas a la implementación de la espectroscopia de infrarrojo cercano (NIRS) como alternativa en la determinación de la calidad de la carne bovina.

Para el cumplimiento del objetivo, se aplicó una revisión de literatura que permitió recuperar información relacionada con la calidad de la carne y su determinación por NIRS. La revisión de literatura se desarrolló siguiendo lo descrito por Snyder (2019). Se utilizó el motor de búsqueda Google Scholar® en el cual se aplicaron fórmulas de búsqueda que incluyeron términos en español e inglés como: "Consumers" + "Consumidor" "NIR", "NIRS", "Beef", "Meat", "Carne", "Bovino", "Beef Quality" y "Calidad de Carne".

\section{Calidad composicional de la carne bovina: aspectos relacionados con las preferencias del consumidor}

Desde el punto de vista zootécnico y de consumo, la carne se define como producto que se obtiene cuando, tras el sacrificio de los bovinos, se somete la canal a maduración durante un periodo, en el que se generan los cambios bioquímicos y estructurales que darán lugar a la transformación del músculo (Paredi et al., 2012), entre los cuales se citan la acidificación, el rigor mortis y la tenderización (Warriss, 2000).

En la carne, los aspectos relacionados con la calidad se pueden definir desde varios escenarios, entre los cuales están la producción, el procesamiento, la distribución y el consumo (Prieto-Benavides, 2006). Desde el punto de vista del consumo, la calidad está determinada por el pago que estaría dispuesto a dar el consumidor por encima del precio medio (Cho et al., 2010) por atributos como: jugosidad, terneza, color, cantidad de grasa, olor e incluso, aspectos éticos relacionados con el proceso de producción y sacrificio de animales (Maltin et al., 2003; Miranda- 
de-la-Lama et al., 2016). Estos atributos se categorizan en intrínsecos y extrínsecos. Los atributos intrínsecos se entienden como aquellas características de la carne que no pueden ser modificadas sin afectar la naturaleza del producto; por su parte, los atributos extrínsecos se asocian a aspectos externos o trasversales a la cadena de producción cárnica (Grunert et al., 2004; Henchion et al., 2017).

Para efectos del relacionamiento con los consumidores, entre los atributos intrínsecos se pueden citar los relacionados con las características de apariencia y de consumo de la carne, mientras que entre los extrínsecos, los inherentes a las características de confianza (Joo et al., 2013).

\section{Atributos de apariencia en la carne}

Dentro de las características de apariencia, las condiciones más importantes para el consumidor son el color de la carne (Mancini \& Hunt, 2005) y su capacidad de retención de agua (Joo et al., 2013), dado que estos atributos generan la primera interacción con el consumidor, mediante la cual, este puede inferir la condición de frescura del producto (Troy \& Kerry, 2010). Se ha demostrado que el consumidor tiende a relacionar atributos de la carne como el color, con características como sabor, terneza, integridad sanitaria, valor nutricional y nivel de satisfacción en la compra (Girolami et al., 2013). Autores como Ardeshiri \& Rose (2018) y Ngapo et al. (2017), han documentado una clara preferencia de los consumidores por diferentes tonalidades de rojo, con mayor frecuencia las tonalidades rojo cereza sobre los colores oscuros o rojos pálidos.

Los atributos de apariencia están relacionados con factores como el tipo de músculo, concentración de hemoglobina y el metabolismo de los músculos. Se indica que el metabolismo de las fibras (glagolítico u oxidativo), pueden afectar características como el pH final de la carne, el color y la terneza, por ejemplo, a través del fenómeno de carne dura, oscura y firme (DFD, por sus siglas en inglés) (Picard et al., 2020); además de factores relacionados con las condiciones previas y posteriores al beneficio del animal y la cadena de custodia del producto cárnico, como el almacenamiento, que influye en la apariencia de la carne (Khan et al., 2015).

Uno de los aspectos determinantes en la decoloración del músculo es la oxidación de la mioglobina (Mancini, 2009), la cual se encuentra, por lo general, en tres formas: como desoximioglobina, la cual está ligada al hierro y no contiene oxígeno, esto genera una apariencia de rojo-púrpura en la carne; le sigue la oxymioglobina, que se genera por adición de oxígeno a la molécula de mioglobina y genera el color rojo cereza que los consumidores asocian con carne fresca y, por último, está la forma de metamioglobina, la cual se alcanza cuando se oxida la molécula de hierro en la mioglobina y la carne toma un color pardo o rojo oscuro, asociado con baja calidad del producto (American Meat Science Association, 2016).

Los estados de oxidaciones de la mioglobina se han relacionado con el consumidor, al respecto, Carpenter et al. (2001) demostraron que tonalidades oscuras en la carne, derivadas de procesos de oxidación de la mioglobina, reciben con mayor frecuencia calificaciones negativas en percepción y preferencia de consumo, con un consecuente efecto negativo en la decisión de compra del producto (Ardeshiri \& Rose, 2018).

En condiciones normales, los músculos con mayor contenido de fibras tipo I (alta demanda metabólica), tienden a decolorarse más rápido por su mayor consumo de oxígeno (Hocquette et al., 2012). Sin embargo, la condición de oscurecimiento de la carne puede alcanzarse de manera acelerada si el animal tuvo un sacrificio inadecuado, con efecto directo en la calidad composicional e instrumental de la carne (Ijaz et al., 2020). Un mal sacrificio se relaciona con alto consumo de las reservas de glucógeno en el músculo por procesos glicolíticos inducidos por

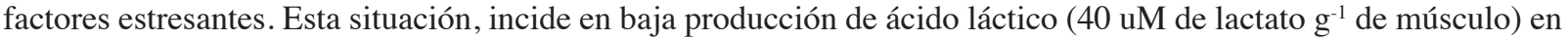
el metabolismo post-morten, que conlleva a niveles de $\mathrm{pH}$ en el músculo superiores al rango óptimo considerado entre 5,4 y 5,7 (Maltin et al., 2003), dando origen a la condición DFD.

Con relación a la pérdida de humedad en la carne, los factores que favorecen esta anomalía se centran en las condiciones pre-sacrificio, y su consecuente respuesta en la disminución rápida del $\mathrm{pH}$, asociado a factores 
ambientales como alta temperatura de almacenamiento (Honikel et al., 1986). La condición de acidificación conduce a la inactividad de las enzimas que intervienen en el proceso de tenderización post-morten (Maltin et al., 2003), que con ayuda de la temperatura, se lleva a la desnaturalización de las proteínas que conforman la estructura muscular (Adzitey \& Nurul, 2011), lo cual favorece la formación de espacios entre las fibras musculares, lo que causa una pérdida excesiva de agua en el músculo (Joo et al., 2013).

\section{Atributos de consumo en la carne}

En el caso de los atributos de consumo, la terneza de la carne se considera como uno de los factores de mayor importancia para los consumidores (Gonzalez \& Phelps, 2018; Hocquette et al., 2020). Para consumidores europeos, se documentó que la terneza de la carne contribuye en un $31 \%$ con la satisfacción de consumo (Liu et al., 2020). Además, se concluyó que, para el caso de consumidores estadounidenses, la terneza de la carne contribuye con un $42 \%$ de la aceptación general (Drey et al., 2017).

Los factores relacionados con la terneza se centran en contenido y la solubilidad del tejido conectivo, la composición y el estado contráctil de las fibras musculares y el grado de rigor mortis (Picard et al., 2020). Se ha reportado que el tejido conectivo, formado en forma predominante por colágeno, varía entre músculos y su concentración depende de la funcionalidad del músculo en la locomoción durante la vida del animal (King et al., 2009). De acuerdo con lo anterior, los músculos ubicados en las extremidades tenderán a presentar mayor dureza que músculos situados en la región dorsal.

Con relación a la terneza, además de la edad, uno de los factores que más incide sobre este atributo de la carne es el proceso de beneficio. Se ha descrito que condiciones de estrés previas al sacrificio, tales como ayunos prolongados, largo período de transporte y ausencia de aturdimiento en el animal, inciden de manera directa en la terneza de la carne, mediadas por el consumo de glucógeno en el músculo previo al sacrificio, el elevado pH final en la carne $(\mathrm{pH}>6)$ y la inactividad de los complejos enzimáticos de resolución del rigor (Ijaz et al., 2020).

\section{Atributos de confianza en la carne}

En la calidad de la carne por atributos de confianza, los consumidores asocian la seguridad y la inocuidad del alimento como los aspectos de mayor importancia (Joo et al., 2013), por ser este un producto alimenticio susceptible a contaminación por patógenos de alto impacto en la salud humana. Entre las posibles causas de contaminación de la carne, se consideran de mayor importancia la ausencia de buenas prácticas de producción en hatos y fincas, la contaminación cruzada de la carne por manejo con aguas contaminadas y trazabilidad en la cadena de frío (Weeranantanaphan et al., 2011).

En los atributos de confianza en la carne, que ganan espacio entre los consumidores, están los aspectos relacionados con la funcionalidad nutricional del alimento y los efectos del consumo de carne en la salud humana (Scollan et al., 2017), así como también, aspectos éticos como el bienestar animal (Miranda-de-Lama et al., 2016), y de responsabilidad ambiental, como los sellos de huella de carbono (Li et al., 2016).

Recientemente, los consumidores se han preocupado por conocer los contenidos nutricionales de los alimentos, en especial la cantidad de nutrientes como la grasa (Lusk, 2019). Para el caso de la carne, varios trabajos han reportado la influencia de la grasa en la modificación de la percepción de los consumidores y preferencias de consumo (Merlino et al., 2018; Meyerding et al., 2018; Ngapo et al., 2017). Este fenómeno se atribuye a la asociación del consumo de carne y el aporte de grasa saturada, con el favorecimiento de enfermedades no trasmisibles como cáncer, diabetes y afecciones cardiacas (Ekmekcioglu et al., 2018; Willett et al., 2019). No obstante, en esta generalización, se desconoce la diferenciación de la composición de la grasa con base en su perfil de ácidos grasos y sus efectos en la salud (Scollan et al., 2014). 
El perfil de ácidos grasos en la carne también se ha relacionado como un factor de interés en la preferencia y decisión de compra por parte de los consumidores. Al respecto, Realini et al. (2014) identificaron la existencia de clúster de consumidores que se inclinaban por el consumo de carne con mayor aporte de ácidos grasos omega 3, caracterizados por mayor conocimiento sobre atributos de la carne. No obstante, se ha demostrado que, aunque los consumidores tienen poco conocimiento del perfil de ácidos grasos en la carne y de su efecto en la salud (BarragánHernández et al., 2021b), la aplicación de esquemas pedagógicos sobre los ácidos grasos poliinsaturados en la carne, favorecen la percepción y preferencia del consumidor por este producto (Flowers, et al., 2018b).

\section{El infrarrojo cercano como alternativa en la determinación de la calidad de la carne}

La espectroscopia en el infrarrojo cercano es una técnica que forma parte del campo de estudio de la espectroscopia molecular, la cual aborda la interacción de la radiación electromagnética con la materia. La técnica se basa en que el espectro lumínico cercano al infrarrojo puede proporcionar información relacionada con los principales elementos estructurales asociados a los compuestos orgánicos, donde los grupos funcionales que corresponden a este espectro son carbono e hidrógeno $(\mathrm{C}-\mathrm{H})$, oxígeno e hidrógeno $(\mathrm{O}-\mathrm{H})$, nitrógeno e hidrógeno $(\mathrm{N}-\mathrm{H})$ y, probablemente, el azufre e hidrógeno $(\mathrm{S}-\mathrm{H})$ y carbono y oxígeno $(\mathrm{C}=\mathrm{O})$ (Workman, 2016).

La base física de la absorción de la luz está relacionada con la naturaleza de los enlaces moleculares, que a su vez son definidos por la unión de átomos en las moléculas. Dependiendo de los enlaces químicos relacionados con los átomos, de la geometría de la molécula y del acoplamiento, se presentan efectos vibracionales en las moléculas (Agelet \& Hurburgh, 2010; Manley \& Baeten, 2018). La interacción de la energía con las moléculas obedece a la ley de Beer-Lambert, que establece que la absorbancia a cualquier longitud de onda es proporcional al número o concentración de moléculas absorbentes presentes en el camino recorrido por la radiación (Workman, 2016).

El proceso de determinación de la calidad de la carne se centra en la obtención de información confiable y precisa, para salvaguardar la integridad del consumidor (Damez \& Clerjon, 2008). Sin embargo, los procesos analíticos asociados a la determinación de la calidad de la carne basados en las técnicas convencionales, en la mayoría de los casos, requieren un equipo particular, consumen tiempo y, para el caso de la determinación composicional, requieren reactivos (Roberts \& Cozzolino, 2016). Estas condiciones limitan la obtención de información de calidad de la carne en tiempo real, el cual es un factor que viene creciendo en la demanda de los consumidores y que, además, es de gran importancia, dada la variabilidad fisicoquímica de este producto (Damez \& Clerjon, 2008).

En la determinación de la calidad composicional de la carne mediante el uso de NIR, se deben tener en cuenta aspectos relacionados con la variabilidad de la muestra de referencia en la característica química a evaluar, la preparación de la muestra y los métodos de captura de espectros que pueden variar de transmitancia a reflectancia (Prieto et al., 2017; Weeranantanaphan et al., 2011). La reflectancia se define como la suma de las radiaciones (difusa y especular) que no son absorbidas por la muestra y, por ende, generan información sobre la composición química del producto. La transmitancia, contiene la radicación que traspasa la muestra y es capturada en detectores en sentido opuesto a la lámpara de emisión del haz monocromático de luz (Alander et al., 2013).

Con relación a los modos de captura de espectros, para la determinación de la calidad composicional de la carne con la tecnología NIR, Mitsumoto \& Ozawa (1991) evaluaron la capacidad de modos transmitancia y reflectancia en la determinación de la calidad de la carne, concluyeron que ambos enfoques generaban una predicción confiable en grasa, energía, humedad y terneza, en muestras de novillos negros japoneses.

La variabilidad en la concentración de las características químicas en la muestra, ha sido descrita como un factor crítico en el éxito en el ajuste de curvas de predicción con NIRS, con efectos negativos, cuando la variación se encuentra en un rango estrecho (Su et al., 2014). La estrecha variabilidad se asociada a un número reducido de datos, lo cual restringiría una adecuada representación de las curvas de calibración para identificar muestras desconocidas (Cozzolino \& Murray, 2002). Se demostró en la determinación de la calidad de carne en muestras de 
músculos provenientes de novillos negros japoneses, que la estrecha variabilidad que presentó el rango de proteína $(5,8 \%)$ frente al rango en la humedad $(16,7 \%)$ y de la grasa $(20,3 \%)$, incidió en el pobre ajuste de la curva de predicción para la determinación de la composición proteica en las muestras (Mitsumoto \& Ozawa, 1991). Se indicó de un pobre ajuste en la determinación de las características de la carne, principalmente en grasa y proteína, que acusa poca variabilidad en el set de muestras para calibración y a poca precisión en las determinaciones analíticas, entre la determinación por Kjendalh (Ripoll et al., 2008), como se puede observar en el Cuadro 1 para atributos de confianza de la carne.

Cuadro 1. Metodologías de evaluación de calidad de carne bovina en sus atributos de confianza y su aplicación mediante Espectroscopía de Infrarrojo Cercano (NIR).

Table 1. Methodologies for evaluating the quality of beef in its trustworthy attributes and its application through near-infrared (NIR) spectroscopy.

\begin{tabular}{|c|c|c|c|c|}
\hline Indicador & Metodología & Referencia & Aplicación de infrarrojo & Referencia NIR \\
\hline \multirow[t]{3}{*}{$\begin{array}{l}\text { Calidad } \\
\text { composicional }\end{array}$} & Kjendal y Soxleth & $\begin{array}{l}\text { AOAC } 981.10 \\
\text { AOAC } 950.46 \\
\text { AOAC } 976.21\end{array}$ & $\begin{array}{c}\checkmark \checkmark \checkmark \text { proteína en muestras de bovino } \\
\text { picada }\left(\mathrm{R}^{2}=0,81, \mathrm{EEP}_{\mathrm{VC}}=21,8\right), \mathrm{y} \\
\otimes \quad \text { para muestras enteras }\left(\mathrm{R}^{2}=0,49,\right. \\
\left.\mathrm{EEP}_{\mathrm{VC}}=23,9\right)\end{array}$ & $\begin{array}{l}\text { Cozzolino \& Murray } \\
\qquad(2002)\end{array}$ \\
\hline & & & $\begin{array}{c}\otimes \text { proteína }\left(\mathrm{R}^{2}=0,11, \mathrm{EEP}=1,019\right) \text { y } \\
\checkmark \checkmark \checkmark \text { grasa }\left(\mathrm{R}^{2}=0,74, \mathrm{EEP}=0,49\right)\end{array}$ & Ripoll et al. (2008) \\
\hline & & & $\begin{array}{l}\checkmark \checkmark \checkmark \text { grasa }\left(R^{2}=0,98, E E P=0,98\right), y \\
\text { en la proteína }\left(R^{2}=0,98, E E P=0,69\right)\end{array}$ & Su et al. (2014) \\
\hline \multirow[t]{3}{*}{$\begin{array}{l}\text { Perfil de ácidos } \\
\text { grasos }\end{array}$} & $\begin{array}{c}\text { Cromatografía de } \\
\text { gases }\end{array}$ & $\begin{array}{l}\text { Angulo et al. } \\
\qquad(2012)\end{array}$ & $\begin{array}{c}\checkmark \checkmark \checkmark \sum \mathrm{SAT} \text { en muestras frescas } \\
\left(\mathrm{R}^{2}=0,98, \mathrm{EEP}_{\mathrm{VC}}=8,15\right), \mathrm{y} \sim \text { para } \\
\text { muestras liofilizadas }\left(\mathrm{R}^{2}=0,67, \mathrm{EEP}_{\mathrm{vC}}\right. \\
=2,46), \text { POLI en muestras frescas } \\
\left(\mathrm{R}^{2}=0,71, \mathrm{EEP}_{\mathrm{vC}}=0,8\right), \text { y en relación } \\
\text { entre } \mathrm{S} / \mathrm{I}\end{array}$ & De-Marchi et al. (2007) \\
\hline & & & $\begin{array}{c}\checkmark \checkmark \checkmark \operatorname{SAT}\left(\mathrm{R}^{2}=0,85, \mathrm{EEP}_{\mathrm{vC}}=0,18\right) \\
\mathrm{y} \otimes \operatorname{para} \text { POLI }\left(\mathrm{R}^{2}=0,25, \mathrm{EEP}_{\mathrm{VC}}\right. \\
=1,16)\end{array}$ & Sierra et al. (2008) \\
\hline & & & $\begin{array}{c}\checkmark \checkmark \checkmark \operatorname{SAT}\left(\mathrm{R}^{2}=0,79, \mathrm{EEP}_{\mathrm{vC}}=0,27\right) \\
\mathrm{y} \sim \operatorname{para} \mathrm{POLI}\left(\mathrm{R}^{2}=0,61, \mathrm{EEP}_{\mathrm{vC}}\right. \\
=0,16)\end{array}$ & Cecchinato et al. (2012) \\
\hline \multirow[t]{3}{*}{$\begin{array}{l}\text { Clasificación o } \\
\text { fraude en carne }\end{array}$} & $\begin{array}{l}\text { PCR, ELISA, } \\
\text { Electroforesis }\end{array}$ & $\begin{array}{c}\text { Doosti et al. } \\
\text { (2014); Bhat et al. } \\
(2015)\end{array}$ & $\begin{array}{c}\checkmark \checkmark \checkmark 85 \% \text { de las muestras para } \\
\text { diferenciar de músculos de cerdo, } \\
\text { oveja, bovino y pollo }\end{array}$ & $\begin{array}{l}\text { Cozzolino \& Murray } \\
\qquad(2004)\end{array}$ \\
\hline & & & $\begin{array}{c}\checkmark \checkmark \checkmark \text { clasificación de carne bovina, } \\
\text { de llama y de caballo }\end{array}$ & $\begin{array}{l}\text { Mamani-Linares et al. } \\
\qquad(2012)\end{array}$ \\
\hline & & & $\begin{array}{c}\checkmark \checkmark \checkmark \text { adulteración de la carne con } \\
\text { adición de grasa en carne congelada }\end{array}$ & Morsy \& Sun (2013) \\
\hline
\end{tabular}

$\checkmark \checkmark \checkmark=$ predicción satisfactoria / Successful prediction.

$\sim=$ predicción aceptable / Acceptable prediction.

$\otimes=$ predicción no satisfactoria / Unsuccessful prediction.

$\sum$ SAT = sumatoria de ácidos grasos saturados / Sum of saturated fatty acids.

$\sum$ POLI = sumatoria de ácidos grasos poliinsaturados / Sum of polyunsaturated fatty acid.

$\mathrm{S} / \mathrm{I}=$ relación saturados - insaturados / saturated - unsaturated ratio.

$\mathrm{R}^{2}=$ coeficiente de determinación / Coefficient of determination.

$\mathrm{EEP}_{\mathrm{vC}}=$ error estándar de la validación cruzada / Standard error of cross-validation.

$\mathrm{EEP}=$ error estándar de la predicción / Standard error of prediction. 
En el caso de la implementación de NIR en la determinación del perfil de ácidos grasos, Sierra et al. (2008), mediante el empleo del modo transmitancia en la cuantificación de ácidos grasos individuales en muestras de longissimus thoracis de bovinos de las razas Asturiana de los Valles y Asturiana de las Montañas, indicaron que la baja concentración individual de los ácidos grasos, asociado al solapamiento de la absorción de varios ácidos grasos en la misma longitud de onda en los grupos moleculares C-H, limitó la capacidad de discriminación y de predicción de las ecuaciones. Esta situación ha generado que los trabajos asociados a perfil de ácidos grasos y su identificación con NIR, sean más exitosos al generar curvas de predicción para ácidos grasos en sumatorias, comparado con predicciones individuales (Ton et al., 2015).

Para el análisis del perfil de ácidos grasos por NIRS, también se han planteado otras alternativas como: a) el enriquecimiento de la variabilidad del set de datos con la inclusión de especies diferentes, como lo reportaron Mourot et al. (2015) al combinar muestras de ovinos y bovinos en un mismo análisis, b) la implementación de alternativas de análisis de los datos, como el aprendizaje de maquinas en la construcción de modelos de predicción (BarragánHernández et al., 2020b) y c) el procesamiento previo de la muestra con liofilización (Giaretta et al., 2019).

Sumado a la poca variabilidad en el set de calibración para las variables de evaluación, es determinante la precisión en las técnicas analíticas convencionales, tal como lo indica Tøgersen et al. (1999), quienes mencionaron que valores altos en el error estándar de los métodos de referencia para la determinación de proteína, el cual representó aproximadamente el 27 \% de la desviación estándar de la muestra, influyeron en forma negativa en la predicción de esta característica a través de NIR. Aspecto discutido por Prieto et al. (2008) y Prevolnik et al. (2010) en algunos atributos de apariencia de la carne.

En el caso de atributos de apariencia, varios autores han demostrado con éxito el uso de NIR (Cuadro 2) para determinar características de la carne que son usadas por los consumidores como primera medida de percepción de calidad, tales como el color y la capacidad de retención de agua, relacionadas en forma directa con el pH (Henchion et al., 2017; Picard et al., 2020).

El color de la carne evaluado en la escala CIELab: $L^{*} a^{*} b^{*}$, relaciona la luminosidad, la intensidad de rojo y la intensidad de amarillo, respectivamente (Mancini \& Hunt, 2005). Se ha demostrado que estos indicadores de color están relacionados con el espectro electromagnético y reaccionan en longitudes de onda asociadas con el rango visible (400 nm -700 nm), en especial para el índice a* y en la región NIR (1230 nm - $1400 \mathrm{~nm}$ y $1600 \mathrm{~nm}-1710$ $\mathrm{nm}$ ) para $L^{*}$ (Prieto et al., 2008). La relación en la intensidad de rojo $\left(a^{*}\right)$ y su alta correlación de absorbancia en el espectro visible, se ha asociado a pigmentos sanguíneos como la mioglobina y sus estados de oxidación (Cozzolino \& Murray, 2002). Para el caso de $L^{*}$, Prieto et al. (2008) indicaron que la absorbancia de este parámetro de color estuvo relacionada con regiones NIR asociadas a enlaces $-\mathrm{CH}$.

Para la capacidad de retención de agua en la carne, autores como Prieto et al. (2008) y De-Marchi et al. (2007), no reportaron relación en los valores espectrales de la región NIR $(1100 \mathrm{~nm}-2500 \mathrm{~nm})$ y argumentaron que la baja precisión en el método de laboratorio empleado como "Gold Standard”, afectó el desarrollo de las ecuaciones de predicción. Contrario a esto, quienes evaluaron cuatro métodos para determinar pérdidas de agua en la carne, determinaron que el método "EZ - pérdidas por goteo", permitió una predicción aceptable de esta característica en las longitudes de onda comprendidas entre $400 \mathrm{~nm}$ y $1100 \mathrm{~nm}$ (Prevolnik et al., 2010). Se ha argumentado que la relación de la pérdida de agua y los valores espectrales, estuvo determinada por otros atributos en la carne como lo son el color y la concentración de grasa.

El tratamiento previo de la muestra se ha identificado como un factor determinante al momento de generar un buen ajuste en las curvas de predicción. Al comparar la implementación de curvas de calibración en muestras de carne picadas y enteras, se indicó que la no homogenización de la muestra (presentación entera) afectó la información obtenida en los espectros por la estructura y organización muscular (Cozzolino \& Murray 2002). Se ha informado que el proceso de liofilización comparado con la muestra de carne fresca, no mejoró la determinación de atributos de consumo como la fuerza de corte (De-Marchi et al., 2007). Sin embargo, los mismos autores sugieren 
Cuadro 2. Metodologías de evaluación de calidad de carne bovina asociados a atributos de apariencia y su aplicación mediante Espectroscopía de Infrarrojo Cercano (NIR).

Table 2. Beef quality evaluation methodologies associated with appearance attributes and their application through near-infrared (NIR) spectroscopy.

\begin{tabular}{|c|c|c|c|c|}
\hline Indicador & Metodología & Referencia & Aplicación de infrarrojo & Referencia NIR \\
\hline \multirow[t]{4}{*}{ Color } & Colorímetro & $\begin{array}{l}\text { American Meat Science } \\
\text { Association (2016) } \\
\text { Girolami et al. (2013); } \\
\text { Mancini \& Hunt (2005) }\end{array}$ & $\begin{array}{l}\checkmark \checkmark \checkmark \text { parámetro } L^{*}\left(\mathrm{R}^{2}=0,85,\right. \\
\left.\operatorname{EEP}_{\mathrm{VC}}=1,16\right)\end{array}$ & Andrés et al. (2007) \\
\hline & & & $\begin{array}{c}\checkmark \checkmark \checkmark \text { parámetro } \mathrm{L}^{*}\left(\mathrm{R}^{2}=0,86, \mathrm{EEP}\right. \\
=1,56) \mathrm{y} \mathrm{b}_{\mathrm{vC}}\left(\mathrm{R}^{2}=0,90, \mathrm{EEP}_{\mathrm{vC}}=1,08\right) \text { en } \\
\text { muestras de bovinos jóvenes }\end{array}$ & Prieto et al. (2008) \\
\hline & & & $\begin{array}{c}\checkmark \checkmark \checkmark \text { parámetro } \mathrm{L}^{*}\left(\mathrm{R}^{2}=0,86, \mathrm{EEP}_{\mathrm{VC}}\right. \\
=0,96), \mathrm{a}^{*}\left(\mathrm{R}^{2}=0,86, \mathrm{EEP}_{\mathrm{vC}}=0,76\right) \mathrm{y} \mathrm{b} * \\
\left(\mathrm{R}^{2}=0,91, \mathrm{EEP}_{\mathrm{VC}}=0,94\right)\end{array}$ & Prieto et al. (2009) \\
\hline & & & $\begin{array}{c}\checkmark \checkmark \checkmark \text { parámetro } \mathrm{L}^{*}\left(\mathrm{R}^{2}=0,82, \mathrm{EEP}_{\mathrm{VC}}\right. \\
=1,91)\end{array}$ & Li et al. (2015) \\
\hline \multirow[t]{4}{*}{$\begin{array}{l}\text { Perdida de } \\
\text { humedad }\end{array}$} & $\begin{array}{l}\text { Pérdidas por goteo } \\
\text { (PPG/CRA) }\end{array}$ & $\begin{array}{l}\text { Honiquel (1998); Juárez } \\
\text { et al. (2012) }\end{array}$ & $\begin{array}{c}\otimes P P G \text { en muestras frescas }\left(\mathrm{R}^{2}=0.10\right. \\
\left.\mathrm{EEP}_{\mathrm{vC}}=3.5\right) \text { y liofilizadas }\left(\mathrm{R}^{2}=0,04\right. \\
\left.\mathrm{EEP}_{\mathrm{vC}}=3,44\right)\end{array}$ & $\begin{array}{l}\text { De-Marchi et al. } \\
\qquad(2007)\end{array}$ \\
\hline & & & $\begin{array}{c}\otimes P P G \text { para músculos en animales } \\
\text { jóvenes y adultos }\left(\mathrm{R}^{2}=0,25, \mathrm{EEP}_{\mathrm{vC}}\right. \\
=0,36)\end{array}$ & Prieto et al. (2008) \\
\hline & & & $\begin{array}{l}\sim \text { CRA en muestras de bovinos } \\
\quad\left(\mathrm{R}^{2}=0,67, \mathrm{EEP}_{\mathrm{vC}}=2,8\right)\end{array}$ & $\begin{array}{l}\text { Rosenvold et al. } \\
\qquad \text { (2009) }\end{array}$ \\
\hline & & & $\sim \mathrm{PPG}\left(\mathrm{R}^{2}=0,58, \mathrm{EEP}_{\mathrm{vC}}=0,91\right)$ & $\begin{array}{l}\text { Prevolnik et al. } \\
\text { (2010) }\end{array}$ \\
\hline \multirow[t]{4}{*}{$\mathrm{pH}$} & Medidor de $\mathrm{pH}$ & $\begin{array}{c}\text { International } \\
\text { Organization for } \\
\text { Standardization (1999); } \\
\text { Young et al. (2004) }\end{array}$ & $\begin{array}{l}\checkmark \checkmark \checkmark \mathrm{pH} \text { en músculo de bovinos } \\
\text { picados }\left(\mathrm{R}^{2}=0,90, \mathrm{EEP}_{\mathrm{vC}}=0,12\right), \mathrm{e} \\
\text { intacto }\left(\mathrm{R}^{2}=0,81, \mathrm{EEP}_{\mathrm{vC}}=0,18\right)\end{array}$ & $\begin{array}{l}\text { Cozzolino y Murray } \\
\text { (2002) }\end{array}$ \\
\hline & & & $\begin{array}{l}\checkmark \checkmark \checkmark \text { pH en muestras de músculo desde } \\
\text { la primera hora y hasta la sexta hora } \\
\text { pos-mortem }\left(\mathrm{R}^{2}=0,85, \mathrm{EEP}_{\mathrm{VC}}=0,20\right)\end{array}$ & $\begin{array}{l}\text { Rosenvold et al. } \\
\qquad \text { (2009) }\end{array}$ \\
\hline & & & $\begin{array}{c}\otimes \text { pH } 7 \text { días post-mortem en bovinos } \\
\text { jóvenes y } 3 \text { días post-morten en } \\
\text { bovinos adultos }\left(\mathrm{R}^{2}=0,41, \mathrm{EEP}_{\mathrm{vC}}\right. \\
=0,06)\end{array}$ & Prieto et al. (2009) \\
\hline & & & $\checkmark \checkmark \checkmark$ pH 45 minutos post-mortem & Li et al. (2015) \\
\hline
\end{tabular}

$\checkmark \checkmark \checkmark=$ predicción satisfactoria / Successful prediction.

$\sim=$ predicción aceptable / Acceptable prediction.

$\otimes=$ predicción no satisfactoria / Unsuccessful prediction .

$\mathrm{R}^{2}=$ coeficiente de determinación / coefficient of determination.

$\mathrm{EEP}_{\mathrm{vC}}=$ error estándar de la validación cruzada / Standard error of cross-validation.

que para la determinación de la grasa intramuscular y del perfil de ácidos grasos, la presentación picada fresca reproduce un mejor ajuste. Se concluyó que la liofilización mejora la predicción de características de la carne por NIRS, se argumenta que la eliminación del agua permite mejor ajuste de predicción (Giaretta et al., 2019). 
Con relación a las caracterices físicas de la carne como terneza y marmóreo, el Cuadro 3, presenta experiencias del uso de infrarrojo cercano. Con relación a la aplicación de la tecnología NIRS en la determinación de las características físicas de la carne, Ripoll et al. (2008) señalaron que procesos previos al análisis como el homogenizado de la muestra, afectan mucho la forma como la luz interactúa con las fibras musculares, que distorsionan el patrón de absorbancia. Este fenómeno influye en forma negativa en la construcción de modelos de predicción, como lo afirmaron Su et al. (2018), quienes reportaron coeficientes de determinación en la validación externa para la terneza medida por Warner-Bratzel entre 0,55 y 0,66 . Sin embargo, no solo el procesamiento de la muestra puede afectar el éxito de la predicción por NIRS en estas características físicas. Se ha demostrado que en muestras intactas también es posible obtener fallas en los modelos predictivos, en especial atribuidos a la baja variabilidad en los datos de referencia, como lo reportaron Magalhães et al. (2018), con coeficientes de determinación en validación externa de 0,02 en marmóreo y 0,4 en Warner-Bratzel.

Cuadro 3. Metodologías de evaluación de calidad de carne bovina en sus atributos de consumo y su aplicación mediante Espectroscopía de Infrarrojo Cercano (NIR).

Table 3. Beef quality evaluation methodologies associated with consumption attributes and their application through near-infrared (NIR) spectroscopy.

\begin{tabular}{|c|c|c|c|c|}
\hline Indicador & Metodología & Referencia & Aplicación de infrarrojo & Referencia NIR \\
\hline \multirow[t]{4}{*}{ Terneza } & $\begin{array}{l}\text { Warner-Bratzler } \\
\text { (WB) }\end{array}$ & $\begin{array}{c}\text { Honiquel (1998); } \\
\text { Damez \& Clerjon } \\
\text { (2008); Juárez et al. } \\
\text { (2012) }\end{array}$ & $\begin{array}{c}\checkmark \checkmark \checkmark \text { terneza con base en la técnica } \\
\text { WB en muestras de bovinos cruzados } \\
\left(\mathrm{R}^{2}=0,74, \mathrm{EEP}_{\mathrm{vC}}=1,08\right)\end{array}$ & Ripoll et al. (2008) \\
\hline & & & $\begin{array}{c}\checkmark \checkmark \checkmark \text { en longissimum thorasic para } \\
\text { la raza Hereford }\left(\mathrm{R}^{2}=0,71, \mathrm{EEP}_{\mathrm{VC}}\right. \\
=28)\end{array}$ & Rosenvold et al. (2009) \\
\hline & & & $\begin{array}{c}\otimes \text { terneza para muestras frescas } \\
\left(\mathrm{R}^{2}=0,08, \mathrm{EEP}_{\mathrm{VC}}=5,21\right) \text { y liofilizadas } \\
\left(\mathrm{R} 2=0,20, \mathrm{EEP}_{\mathrm{VC}}=4,99\right)\end{array}$ & De-Marchi et al. (2007) \\
\hline & & & $\begin{array}{c}\checkmark \checkmark \checkmark \text { terneza en longissimus dorsi } \\
\text { de bovinos mestizos }\left(\mathrm{R}^{2}=0,85, \mathrm{EEP}_{\mathrm{vC}}\right. \\
=3,9)\end{array}$ & de-Olivera (2014) \\
\hline \multirow[t]{4}{*}{ Marmóreo } & $\begin{array}{l}\text { Método subjetivo, } \\
\text { panel evaluador }\end{array}$ & $\begin{array}{l}\text { American Meat Science } \\
\text { Association (2016); } \\
\text { Association of Official } \\
\text { Agricultural Chemist } \\
\text { (2000) }\end{array}$ & $\begin{array}{c}\checkmark \checkmark \checkmark \text { GIM en muestras de musculo } \\
\text { en bovino picadas }\left(\mathrm{R}^{2}=0,96, \mathrm{EEP}_{\mathrm{vC}}\right. \\
=44,8) \text { y enteras }\left(\mathrm{R}^{2}=0,89, \mathrm{EEP}_{\mathrm{vC}}\right. \\
=46,9)\end{array}$ & $\begin{array}{l}\text { Cozzolino \& Murray } \\
\text { (2002) }\end{array}$ \\
\hline & & & $\begin{array}{c}\checkmark \checkmark \checkmark \text { clasificaciones de canales } \\
\quad\left(\mathrm{R}^{2}=0,82, \mathrm{EEP}_{\mathrm{VC}}=0,44\right)\end{array}$ & Alomar et al. (2003) \\
\hline & & & $\begin{array}{l}\checkmark \checkmark \checkmark \text { GIM en músculo de bovino } \\
\text { picado }\left(\mathrm{R}^{2}=0,98, \mathrm{EEP}_{\mathrm{vC}}=0,39\right)\end{array}$ & Prevolnik et al. (2010) \\
\hline & & & $\begin{array}{c}\checkmark \checkmark \checkmark \text { GIM en músculo de bovino } \\
\text { picado }\left(\mathrm{R}^{2}=0,81, \mathrm{EEP}_{\mathrm{VC}}=0,31\right)\end{array}$ & Cecchinato et al. (2012) \\
\hline
\end{tabular}

$\checkmark \checkmark \checkmark=$ predicción satisfactoria / Successful prediction.

$\otimes=$ predicción no satisfactoria / Unsuccessful prediction.

GIM = grasa intramuscular / Intramuscular fat.

$\mathrm{R}^{2}=$ coeficiente de determinación / coefficient of determination.

$\mathrm{EEP}_{\mathrm{vC}}=$ error estándar de la validación cruzada / Standard error of cross-validation. 


\section{Otras aplicaciones del NIRS en la industria cárnica}

Además de la determinación de los atributos de calidad en la carne, la tecnología NIRS tiene otras aplicaciones importantes en la industria cárnica bovina que permiten desarrollar procesos de determinación, autenticación y anticipación de características o del sistema de producción que mejoran la calidad del producto (Prieto et al., 2017; Roberts \& Cozzolino, 2016).

La presencia de la anomalía DFD, derivada de la alteración en el pH de la carne, como fue descrito, constituye una importante fuente de pérdida de valor de canal en la industria cárnica (Ijaz et al., 2020). La determinación de carne DFD con métodos rápidos y confiables, permiten a la industria valorar en forma objetiva la presencia de esta anomalía (Prieto et al., 2018). Con relación a las experiencias descritas en la literatura, Prieto et al. (2014) demostraron que la aplicación de NIRS, logra discriminar la presencia de carne DFD en un $95 \%$ de los casos evaluados, lo cual representa una herramienta precisa y confiable para la industria.

Otra de las grandes aplicaciones en las que se ha avanzado con el uso de NIRS en la industria cárnica, es la determinación de características de la carne en plantas de beneficio (Løvland \& Wold, 2020). Se recopilaron diferentes experiencias en la aplicación de la tecnología NIRS en la medición de características de la carne sobre la carcasa y se documentaron casos exitosos en la cuantificación de grasa, proteína y sumatorias de ácidos grasos (Chapman et al., 2020). Se han reportado que para atributos físicos de la carne como el color $\left(a^{*}, b^{*}, C^{*}\right.$ y $\left.H^{*}\right)$, pérdidas por goteo, pérdidas por cocción y fuerza de corte, la determinación por NIRS en la línea de proceso aún debe ser perfeccionada, puesto que los coeficientes de determinación en la validación varían de 0,19 a 0,6, lo cual indica baja habilidad predictiva (Savoia et al., 2020). Contrario a lo anterior, los mismos autores afirmaron que para la variable luminosidad en el color $\left(L^{*}\right)$, se obtuvo un modelo con un coeficiente de determinación en la validación de 0,8 .

El desarrollo de equipos NIRS portátiles y sensores de menor tamaño, han permito el avance de investigaciones en línea de beneficio, que abarcan la posibilidad de medir, además de los parámetros clásicos de la calidad de la carne, contenidos nutricionales como minerales. La investigación desarrollada por Patel et al. (2020), demostró que se pueden desarrollar modelos predictivos para fósforo $\left(R^{2}\right.$ validación 0,78$)$ y hierro $\left(R^{2}\right.$ validación 0,81$)$ en la carne.

La autenticación de la carne como herramienta de preservación de nichos de mercado o valoración de atributos diferenciales del producto, ya sea por condición geográfica o fisicoquímica, ha sido otra de las grandes ventajas de la aplicación del NIRS en la industria de alimentos (Cozzolino, 2016). El trabajo desarrollado por BarragánHernández et al. (2021a), demostró que la aplicación del NIRS permite diferenciar la carne procedente de sistemas de producción que utilizan cebada, versus los que usan maíz en los sistemas de finalización de carne en la región de Alberta en Canadá. Esta diferenciación permite preservar el sistema de producción con cebada, junto con su ámbito socio-cultural en los productores, frente a posibles amenazas de cambio en el entorno productivo regional.

También se ha abordado el uso de la tecnología NIRS para anticipar la percepción del consumidor en algunos atributos de la carne y la disposición a compra. La aplicación de NIRS por trasmitancia, permitió discriminar la disposición de compra de los consumidores en ocho de cada diez muestras evaluadas en la validación externa. Este resultado permite anticipar la probabilidad que tiene la carne de ser comprada y definir si puede ser enviada a góndola o usada en la producción de subproductos (Barragán-Hernández et al., 2020a).

\section{Conclusiones}

La necesidad de conocer los atributos de calidad de la carne bovina por parte de los consumidores, demanda la implementación de sistemas de evaluación rápidos y confiables. 
La tecnología de infrarrojo cercano ha sido desarrollada para su uso en la identificación de los atributos de apariencia, consumo y confianza en la carne bovina. Se ha demostrado que la implementación de NIRS, dinamizaría la capacidad de respuesta a los consumidores y permitiría explorar el acceso a pago de producto por calidad y/o información relacionada con elementos funcionales en la carne.

Los atributos de mayor relación con los enlaces orgánicos que se absorben en el espectro de luz electromagnético, pueden ser medidos con la tecnología NIRS. En algunos casos, con ajustes metodológicos como el procesamiento de la muestra y el tipo de equipo usado (reflectancia y trasmitancia). Por el contrario, las características físicas de la carne aún demuestran mucha heterogeneidad en su respuesta, por lo cual es necesario explorar más aplicaciones con NIRS.

En términos de aplicabilidad en la industria, se abordaron aplicaciones recientes y novedosas de la tecnología NIRS en la industria cárnica, que van desde la determinación de anomalías en la carne y contenidos de minerales, hasta la autenticación de sistemas de producción y anticipación de la disposición de compra de los consumidores. Estas aplicaciones se vienen abriendo paso en la industria y en la medida que el desarrollo tecnológico permita la disminución de los costos de los equipos NIRS, se masificará la adopción de estas tecnologías en las plantas de beneficio bovino. En adición, las tecnologías de bajo impacto ambiental como las planteadas en esta revisión, son las llamadas a participar en la industria cárnica, toda vez que minimizan la producción de residuos contaminantes como se presentan en las técnicas de referencia y ayudan a minimizar el impacto ambiental de la cadena de producción de la carne.

\section{Agradecimientos}

Los autores agradecen especialmente al Ministerio de Ciencia y Tecnología de Colombia por el apoyo brindado en la convocatoria de doctorado nacional 727 de 2015; así como también, a la estrategia de sostenibilidad de los grupos de investigación GRICA y Biogénesis de la Universidad de Antioquia y a la Corporación Colombiana de Investigación Agropecuaria (Agrosavia).

\section{Referencias}

Aboah, J., \& Lees, N. (2020). Consumers use of quality cues for meat purchase: Research trends and future pathways. Meat Science, 166(April), Article 108142. https://doi.org/10.1016/j.meatsci.2020.108142

Adzitey, F., \& Nurul, H. (2011). Pale soft exudative (PSE) and dark firm dry (DFD) meats: Causes and measures to reduce these incidences - a mini review. International Food Research Journal, 18(1), 11-20.

Agelet, L. E., \& Hurburgh, C. R. (2010). A tutorial on near infrared spectroscopy and its calibration. Critical Reviews in Analytical Chemistry, 40(4), 246-260. https://doi.org/10.1080/10408347.2010.515468

Alander, J. T., Bochko, V., Martinkauppi, B., Saranwong, S., \& Mantere, T. (2013). A review of optical nondestructive visual and Near-Infrared methods for food quality and safety. International Journal of Spectroscopy, 2013, Article 341402. https://doi.org/10.1155/2013/341402

Alomar, D., Gallo, C., Castañeda, M., Fuchslocher, R., Castaneda, M., \& Fuchslocher, R. (2003). Chemical and discriminant analysis of bovine meat by near infrared reflectance spectroscopy (NIRS). Meat Science, 63(4), 441-450. https://doi. org/10.1016/S0309-1740(02)00101-8 
American Meat Science Association. (2016). Research guidelines for cookery, sensory evaluation, and instrumental tenderness measurements of meat. American Meat Science Association. https://doi.org/10.1590/s0103-84782012000400025

Andrés, S., Murray, I., Navajas, E. A., Fisher, A. V., Lambe, N. R., \& Bünger, L. (2007). Prediction of sensory characteristics of lamb meat samples by near infrared reflectance spectroscopy. Meat Science, 76(3), 509-516. https://doi.org/10.1016/j. meatsci.2007.01.011

Angulo, J., Nürnberg, K., Mahecha, L., Olivera, M., \& Dannenberger, D. (2012). Manual of lipid extraction, methylation and gas chromatography, for the study of different tissues in ruminant research. Biogénesis. https://revistas.udea.edu.co/ index.php/biogenesis/article/view/326020/20783311

Ardeshiri, A., \& Rose, J. M. (2018). How Australian consumers value intrinsic and extrinsic attributes of beef products. Food Quality and Preference, 65(October), 146-163. https://doi.org/10.1016/j.foodqual.2017.10.018

Arenas-De-Moreno, L., Jerez-Timaure, N., Valerio-Hernández, J., Huerta-Leidenz, N. O., \& Rodas-González, A. (2020). Attitudinal determinants of beef consumption in Venezuela: A retrospective survey. Foods, 9, Article 202. https://doi. org/10.3390/foods 9020202

Association of Official Agricultural Chemists (2000). Fat (crude) or ether extract in meat 960.39-1960. AOAC Int.

Bhat, M. M., Jalal, H., Para, P. A., Bukhari, S. A., Ganguly, S., Bhat, A. A., Wakchaure, R., \& Qadri, K. (2015). Fraudulent adulteration / Substitution of meat: A review. Journal of Recent Research and Applied Studies, 2(12), 21-33.

Barragán-Hernández, W., Aalhus, J. L., Penner, G., Dugan, M. E. R., Juárez, M., López-Campos, Ó., Vahmani, P., Segura, J., Angulo, J., \& Prieto, N. (2021a). Authentication of barley-finished beef using visible and near infrared spectroscopy (Vis-NIRS) and different discrimination approaches. Meat Science, 172, Article 108342. https://doi.org/10.1016/j. meatsci.2020.108342

Barragán-Hernández, W., Mahecha-Ledesma, L., Angulo-Arizala, J., \& Olivera-Angel, M. (2020a). Near-infrared spectroscopy as a beef quality tool to predict consumer acceptance. Foods, 9(8), 1-15. https://doi.org/10.3390/foods9080984

Barragán Hernández, W. A., Mahecha-Ledesma, L., Burgos-Paz, W. O., Olivera-Angel, M. M., \& Angulo-Arizala, J. (2020b). Using near-infrared spectroscopy to determine intramuscular fat and fatty acids of beef applying different prediction approach. Journal of Animal Science, 98(11), Article 342. https://doi.org/10.1093/jas/skaa342

Barragán-Hernández, W., Mahecha-Ledesma, L., Olivera-Angel, M., \& Angulo-Arizala, J. (2021b). ¿Cómo los consumidores valoran atributos de calidad de carne bovina y su disposición a pago? Revista Biotecnología en el Sector Agropecuario y Agroindustrial, 19(1), 1-15.

Carpenter, C. E., Cornforth, D. P., \& Whittier, D. (2001). Consumer preferences for beef color and packaging did not affect eating satisfaction. Meat Science, 57(4), 359-363. https://doi.org/10.1016/S0309-1740(00)00111-X

Cecchinato, A., de Marchi, M., Penasa, M., Casellas, J., Schiavon, S., \& Bittante, G. (2012). Genetic analysis of beef fatty acid composition predicted by near-infrared spectroscopy. Journal of Animal Science, 90(2), 429-438. https://doi. org/10.2527/jas.2011-4150

Chapman, J., Elbourne, A., Truong, V. K., \& Cozzolino, D. (2020). Shining light into meat - a review on the recent advances in in vivo and carcass applications of near infrared spectroscopy. International Journal of Food Science and Technology, 55(3), 935-941. https://doi.org/10.1111/ijfs.14367 
Cho, S. H., Kim, J., Park, B. Y., Seong, P. N., Kang, G. H., Kim, J. H., Jung, S. G., Im, S. K., \& Kim, D. (2010). Assessment of meat quality properties and development of a palatability prediction model for Korean Hanwoo steer beef. Meat Science, 86(1), 236-242. https://doi.org/10.1016/j.meatsci.2010.05.011

Cocking, C., Walton, J., Kehoe, L., Cashman, K. D., \& Flynn, A. (2020). The role of meat in the European diet: current state of knowledge on dietary recommendations, intakes and contribution to energy and nutrient intakes and status. Nutrition Research Reviews, 33(2), 181-189. https://doi.org/10.1017/S0954422419000295

Cozzolino, D. (2016). 7 - Near infrared spectroscopy and food authenticity. In M. Espiñeira, \& F. J. Santaclara (Eds.), Advances in Food Traceability Techniques and Technologies (pp. 119-136). Woodhead Publishing. https://doi.org/10.1016/ B978-0-08-100310-7.00007-7

Cozzolino, D., \& Murray, I. (2002). Effect of sample presentation and animal muscle species on the analysis of meat by near infrared reflectance spectroscopy. Journal of Near Infrared Spectroscopy, 10(1), 37-44. https://doi.org/10.1255/ jnirs.319

Cozzolino, D., \& Murray, I. (2004). Identification of animal meat muscles by visible and near infrared reflectance spectroscopy. LWT - Food Science and Technology, 37(4), 447-452. https://doi.org/10.1016/j.lwt.2003.10.013

Damez, J. L., \& Clerjon, S. (2008). Meat quality assessment using biophysical methods related to meat structure. Meat Science, 80(1), 132-149. https://doi.org/10.1016/j.meatsci.2008.05.039

De-Marchi, M., Berzaghi, P., Boukha, A., Mirisola, M., \& Gallo, L. (2007). Use of near infrared spectroscopy for assessment of beef quality traits. Italian Journal of Animal Science, 6(Suppl. 1), 421-423. https://doi.org/10.4081/ijas.2007.1s.421

de-Olivera, R., (2014). Modelos de calibração multivariada por NIRS para a predição de características de qualidade da carne bovina [Tese de Doutorado, não publicada]. Universidade Federal de Goiás.

Doosti, A., Ghasemi Dehkordi, P., \& Rahimi, E. (2014). Molecular assay to fraud identification of meat products. Journal of Food Science and Technology, 51(1), 148-152. https://doi.org/10.1007/s13197-011-0456-3

Drey, L., Legako, J., Brooks, J., Miller, M., \& O’quinn, T. (2017). The contribution of tenderness, juiciness, and flavor to overall consumer beef eating experience. Meat and Muscle Biology, 1(3), 13-13. https://doi.org/10.221751/rmc2017.012

Ekmekcioglu, C., Wallner, P., Kundi, M., Weisz, U., Haas, W., \& Hutter, H. P. (2018). Red meat, diseases, and healthy alternatives: A critical review. Critical Reviews in Food Science and Nutrition, 58(2), 247-261. https://doi.org/10.10 80/10408398.2016.1158148

Flowers, S., Hamblen, H., Leal-Gutiérrez, J. D., Elzo, M. A., Johnson, D. D., \& Mateescu, R. G. (2018a). Fatty acid profile, mineral content, and palatability of beef from a multibreed Angus-Brahman population. Journal of Animal Science, 96(10), 4264-4275. https://doi.org/10.1093/jas/sky300

Flowers, S., McFadden, B., Carr, C., \& Mateescu, R. (2018b). Understanding beef nutritional attributes contributes to consumers' willingness-to-pay for a healthier product. Meat and Muscle Biology, 2(2), 15-16. https://doi.org/10.221751/ rmc2018.014

Flowers, S., McFadden, B. R., Carr, C. C., \& Mateescu, R. G. (2019). Consumer preferences for beef with improved nutrient profile. Journal of Animal Science, 97(12), 4699-4709. https://doi.org/10.1093/jas/skz327 
Font-i-Furnols, M., Fulladosa, E., Prevolnik Povše, M., \& Čandek-Potokar, M. (2015). Future trends in non-invasive technologies suitable for quality determinations In M. Font-i-Furnols, M. Čandek-Potokar, C. Maltin, \& M. Prevolnik Povše (Eds.), A Handbook of Reference Methods for Meat Quality Assessment (pp. 90-104). European Cooperation in Science and Technology.

Font-i-Furnols, M., \& Guerrero, L. (2014). Consumer preference, behavior and perception about meat and meat products: An overview. Meat Science, 98(3), 361-371. https://doi.org/10.1016/j.meatsci.2014.06.025

Giaretta, E., Mordenti, A., Palmonari, A., Brogna, N., Canestrari, G., Belloni, P., Cavallini, D., Mammi, L., Cabbri, R., \& Formigoni, A. (2019). NIRs calibration models for chemical composition and fatty acid families of raw and freezedried beef: a comparison. Journal of Food Composition and Analysis, 83, Article 103257. https://doi.org/10.1016/j. jfca.2019.103257

Girolami, A., Napolitano, F., Faraone, D., \& Braghieri, A. (2013). Measurement of meat color using a computer vision system. Meat Science, 93(1), 111-118. https://doi.org/10.1016/j.meatsci.2012.08.010

Gonzalez, J. M., \& Phelps, K. J. (2018). United States beef quality as chronicled by the national beef quality audits, beef consumer satisfaction projects, and national beef tenderness surveys - A review. Asian-Australasian Journal of Animal Sciences, 31(7), 1036-1042. https://doi.org/10.5713/ajas.18.0199

Grunert, K. G., Bredahl, L., \& Bruns $\varnothing$, K. (2004). Consumer perception of meat quality and implications for product development in the meat sector - A review. Meat Science, 66(2), 259-272. https://doi.org/10.1016/S0309-1740(03)00130-X

Henchion, M. M., McCarthy, M., \& Resconi, V. C. (2017). Beef quality attributes: A systematic review of consumer perspectives. Meat Science, 128, 1-7. https://doi.org/10.1016/j.meatsci.2017.01.006

Hocquette, J. F., Cassar-Malek, I., Jurie, C., Bauchart, D., Picard, B., \& Renand, G. (2012). Relationships between muscle growth potential, intramuscular fat content and different indicators of muscle fibre types in young Charolais bulls. Animal Science Journal, 83(11), 750-758. https://doi.org/10.1111/j.1740-0929.2012.01021.x

Hocquette, J. F., Ellies-Oury, M. P., Legrand, I., Pethick, D., Gardner, G., Wierzbicki, J., \& Polkinghorne, R. J. (2020). Research in beef tenderness and palatability in the era of Big Data. Meat and Muscle Biology, 4(2), Article 9488. https://doi. org/10.22175/mmb. 9488

Honikel, K. O. (1998). Reference methods for the assessment of physical characteristics of meat. Meat Science, 49(4), $447-457$. https://doi.org/10.1016/S0309-1740(98)00034-5

Honikel, K. O., Kim, C. J., Hamm, R., \& Roncales, P. (1986). Sarcomere shortening of prerigor muscles and its influence on drip loss. Meat Science, 16(4), 267-282. https://doi.org/10.1016/0309-1740(86)90038-0

International Organization for Standardization (1999). Meat and meat products: Measurement of pH 2917:1999. ISO.

Ijaz, M., Li, X., Zhang, D., Hussain, Z., Ren, C., Bai, Y., \& Zheng, X. (2020). Association between meat color of DFD beef and other quality attributes. Meat Science, 161, Article 107954. https://doi.org/10.1016/j.meatsci.2019.107954

Joo, S. T., Kim, G. D., Hwang, Y. H., \& Ryu, Y. C. (2013). Control of fresh meat quality through manipulation of muscle fiber characteristics. Meat Science, 95(4), 828-836. https://doi.org/10.1016/j.meatsci.2013.04.044

Joseph, P., Searing, A., Watson, C., \& McKeague, J. (2020). Alternative proteins: Market research on consumer trends and emerging landscape. Meat and Muscle Biology, 4(2), Article 16. https://doi.org/10.22175/mmb.11225 
Juárez, M., Aldai, N., López-Campos, Ó., Dugan, M. E. R., Uttaro, B., \& Aalhus, J. L. (2012). Beef texture and juiciness In Y. H. Hui (Ed.), Handbook of meat and processing (2 ${ }^{\text {nd }}$ Ed., pp. 177-206). CRC Press. https://doi.org/10.1201/b11479-13

Khan, M. I., Jo, C., \& Tariq, M. R. (2015). Meat flavor precursors and factors influencing flavor precursors-A systematic review. Meat Science, 110, 278-284. https://doi.org/10.1016/j.meatsci.2015.08.002

King, D. A., Wheeler, T. L., Shackelford, S. D., \& Koohmaraie, M. (2009). Fresh meat texture and tenderness. In J. P. Kerry, \& D. Ledward (Eds.), Improving the sensory and nutritional quality of fresh meat (pp. 61-88). Woodhead Publishing Limited. https://doi.org/10.1533/9781845695439.1.61

Li, X., Feng, F., Gao, R., Wang, L., Qian, Y., Li, C., \& Zhou, G. (2015). Application of near infrared reflectance (NIR) spectroscopy to identify potential PSE meat. Journal of the Science of Food and Agriculture, 96(9), 3148-3156. https://doi.org/10.1002/jsfa.7493

Li, X., Jensen, K. L., Clark, C. D., \& Lambert, D. M. (2016). Consumer willingness to pay for beef grown using climate friendly production practices. Food Policy, 64, 93-106. https://doi.org/10.1016/j.foodpol.2016.09.003

Liu, J., Ellies-Oury, M. P., Chriki, S., Legrand, I., Pogorzelski, G., Wierzbicki, J., Farmer, L., Troy, D., Polkinghorne, R., \& Hocquette, J. F. (2020). Contributions of tenderness, juiciness and flavor liking to overall liking of beef in Europe. Meat Science, 168(April), Article 108190. https://doi.org/10.1016/j.meatsci.2020.108190

Løvland, A., \& Wold, J. P. (2020). NIR spectroscopic techniques for quality and process control in the meat industry. Meat and Muscle Biology, 4(2), Article 10020. https://doi.org/10.22175/mmb.10020

Lusk, J. L. (2019). Consumer beliefs about healthy foods and diets. PLoS ONE, 14(10), Article e0223098. https://doi. org/10.1371/journal.pone.0223098

Magalhães, A. F., Teixeira, G. H., Ríos, A. C., Silva, D. B., Mota, L. F., Muniz, M. M., de-Morais, C. de L., de-Lima, K. M., Júnior, L. C., Baldi, F., Carvalheiro, R., de-Oliveira, H., Chardulo, L. A., \& de-Albuquerque, L. G. (2018). Prediction of meat quality traits in Nelore cattle by near-infrared reflectance spectroscopy. Journal of Animal Science, 96(10), 4229-4237. https://doi.org/10.1093/jas/sky284

Maltin, C., Balcerzak, D., Tilley, R., \& Delday, M. (2003). Determinants of meat quality: tenderness. Proceedings of the Nutrition Society, 62, 337-347. https://doi.org/10.1079/PNS2003248

Mamani-Linares, L. W., Gallo, C., \& Alomar, D. (2012). Identification of cattle, llama and horse meat by near infrared reflectance or transflectance spectroscopy. Meat Science, 90(2), 378-385. https://doi.org/10.1016/j.meatsci.2011.08.002

Mancini, R. A. (2009). Meat color. In J. P. Kerry, \& D. Ledward (Eds.), Improving the sensory and nutritional quality of fresh meat (pp. 89-110). Woodhead Publishing Limited. https://doi.org/10.1533/9781845695439.1.89

Mancini, R. A., \& Hunt, M. C. (2005). Current research in meat color. Meat Science, 71(1), 100-121. https://doi.org/10.1016/j. meatsci.2005.03.003

Manley, M., \& Baeten, V. (2018). Spectroscopic technique: Near Infrared Spectroscopy (NIR). In D. W. Sun (Ed.), Modern Techniques for Food Authentication (2 ${ }^{\text {nd }}$ ed., pp. 51-102). Elsevier Inc. https://doi.org/10.1016/b978-0-12-8142646.00003-7

McCarthy, S. N., Henchion, M., White, A., Brandon, K., \& Allen, P. (2017). Evaluation of beef eating quality by Irish consumers. Meat Science, 132(April), 118-124. https://doi.org/10.1016/j.meatsci.2017.05.005 
Merlino, V. M., Borra, D., Girgenti, V., Dal Vecchio, A., \& Massaglia, S. (2018). Beef meat preferences of consumers from Northwest Italy: Analysis of choice attributes. Meat Science, 143(September), 119-128. https://doi.org/10.1016/j. meatsci.2018.04.023

Meyerding, S. G. H., Gentz, M., Altmann, B., \& Meier-Dinkel, L. (2018). Beef quality labels: A combination of sensory acceptance test, stated willingness to pay, and choice-based conjoint analysis. Appetite, 127, 324-333. https://doi. org/10.1016/j.appet.2018.05.008

Miller, R. (2020). Drivers of consumer liking for beef, pork, and lamb: A review. Foods, 9(4), Article 428. https://doi. org/10.3390/foods 9040428

Miranda-de-la-Lama, G. C., Estévez-Moreno, L. X., Sepúlveda, W. S., Estrada-Chavero, M. C., Rayas-Amor, A. A., Villarroel, M., \& María, G. A. (2016). Mexican consumers' perceptions and attitudes towards farm animal welfare and willingness to pay for welfare friendly meat products. Meat Science, 125, 106-113. https://doi.org/10.1016/j.meatsci.2016.12.001

Mitsumoto, M., \& Ozawa, S. (1991). Near-infrared spectroscopy determination of physical and chemical characteristics in beef cuts. Journal of Food Science 56(6), 1493-1496. https://doi.org/10.1111/j.1365-2621.1991.tb08623.x

Morsy, N., \& Sun, D. W. (2013). Robust linear and non-linear models of NIR spectroscopy for detection and quantification of adulterants in fresh and frozen-thawed minced beef. Meat Science, 93(2), 292-302. https://doi.org/10.1016/j. meatsci.2012.09.005

Mourot, B. P., Gruffat, D., Durand, D., Chesneau, G., Mairesse, G., \& Andueza, D. (2015). Breeds and muscle types modulate performance of near-infrared reflectance spectroscopy to predict the fatty acid composition of bovine meat. Meat Science, 99, 104-112. https://doi.org/10.1016/j.meatsci.2014.08.014

Ngapo, T. M., Braña Varela, D., \& Rubio-Lozano, M. S. (2017). Mexican consumers at the point of meat purchase. Beef choice. Meat Science, 134, 34-43. https://doi.org/10.1016/j.meatsci.2017.07.013

Paredi, G., Raboni, S., Bendixen, E., de Almeida, A. M., \& Mozzarelli, A. (2012). "Muscle to meat" molecular events and technological transformations: The proteomics insight. Journal of Proteomics, 75(14), 4275-4289. https://doi. org/10.1016/j.jprot.2012.04.011

Patel, N., Toledo-Alvarado, H., Cecchinato, A., \& Bittante, G. (2020). Predicting the content of 20 minerals in beef by different portable near-infrared (NIR) spectrometers. Foods, 9(10), Article 1389. https://doi.org/10.3390/foods9101389

Picard, B., Gagaoua, M., \& Gagaoua, M. (2020). Muscle fiber properties in cattle and their relationships with meat qualities: An overview. Journal of Agricultural and Food Chemistry, 68(22), 6021-6039. https://doi.org/10.1021/acs.jafc.0c02086

Prevolnik, M., Candek-Potokar, M., \& Škorjanc, D. (2010). Predicting pork water-holding capacity with NIR spectroscopy in relation to different reference methods. Journal of Food Engineering, 98(3), 347-352. https://doi.org/10.1016/j. jfoodeng.2009.11.022

Prieto, N., Andrés, S., Giráldez, F. J., Mantecón, A. R., \& Lavín, P. (2008). Ability of near infrared reflectance spectroscopy (NIRS) to estimate physical parameters of adult steers (oxen) and young cattle meat samples. Meat Science, 79(4), 692-699. https://doi.org/10.1016/j.meatsci.2007.10.035

Prieto, N., Ross, D. W., Navajas, E. A., Nute, G. R., Richardson, R. I., Hyslop, J. J., \& Roehe, R. (2009). On-line application of visible and near infrared reflectance spectroscopy to predict chemical-physical and sensory characteristics of beef quality. Meat Science, 83(1), 96-103. https://doi.org/10.1016/j.meatsci.2009.04.005 
Prieto, N., López-Campos, Suman, S. P., Uttaro, B., Rodas-González, A., \& Aalhus, J. L. (2018). Exploring innovative possibilities of recovering the value of dark-cutting beef in the Canadian grading system. Meat Science, 137, 77-84. https://doi.org/10.1016/j.meatsci.2017.11.013

Prieto, N, López-Campos, Ó., Zijlstra, R. T., Uttaro, B., \& Aalhus, J. L. (2014). Discrimination of beef dark cutters using visible and near infrared reflectance spectroscopy. Canadian Journal of Animal Science, 94(3), 445-454. https://doi. org/10.4141/cjas-2014-024

Prieto, N, Pawluczyk, O., Dugan, M. E. R., \& Aalhus, J. L. (2017). A review of the principles and applications of near-infrared spectroscopy to characterize meat, fat, and meat products. Applied Spectroscopy, 71(7), 1403-1426. https://doi. org/10.1177/0003702817709299

Prieto-Benavides, N. (2006). Aplicación de la tecnología NIRS para estimar parámetros indicativos de la calidad de la carne de vacuno [Disertación Doctoral, Universidad de León]. Respositorio del Consejo Superior de Investigaciones Científicas. http://digital.csic.es/handle/10261/21982

Realini, C. E., Kallas, Z., Pérez-Juan, M., Gómez, I., Olleta, J. L., Beriain, M. J., Albertí, P., \& Sañudo, C. (2014). Relative importance of cues underlying Spanish consumers' beef choice and segmentation, and consumer liking of beef enriched with n-3 and CLA fatty acids. Food Quality and Preference, 33, 74-85. https://doi.org/10.1016/j. foodqual.2013.11.007

Ripoll, G., Albertí, P., Panea, B., Olleta, J. L., \& Sañudo, C. (2008). Near-infrared reflectance spectroscopy for predicting chemical, instrumental and sensory quality of beef. Meat Science, 80(3), 697-702. https://doi.org/10.1016/j. meatsci.2008.03.009

Roberts, J. J., \& Cozzolino, D. (2016). An overview on the application of chemometrics in food science and technology-An approach to quantitative data analysis. Food Analytical Methods, 9(12), 3258-3267. https://doi.org/10.1007/s12161016-0574-7

Rosenvold, K., Micklander, E., Hansen, P. W., Burling-Claridge, R., Challies, M., Devine, C., \& North, M. (2009). Temporal, biochemical and structural factors that influence beef quality measurement using near infrared spectroscopy. Meat Science, 82(3), 379-388. https://doi.org/10.1016/j.meatsci.2009.02.010

Sakadevan, K., \& Nguyen, M. L. (2017). Livestock production and its impact on nutrient pollution and greenhouse gas emissions. Advances in Agronomy, 141, 147-184. https://doi.org/10.1016/bs.agron.2016.10.002

Savoia, S., Albera, A., Brugiapaglia, A., Di Stasio, L., Ferragina, A., Cecchinato, A., \& Bittante, G. (2020). Prediction of meat quality traits in the abattoir using portable and hand-held near-infrared spectrometers. Meat Science, 161(March), Article 108017. https://doi.org/10.1016/j.meatsci.2019.108017

Scollan, N. D., Dannenberger, D., Nuernberg, K., Richardson, I., MacKintosh, S., Hocquette, J. F., \& Moloney, A. P. (2014). Enhancing the nutritional and health value of beef lipids and their relationship with meat quality. Meat Science, 97(3), 384-394. https://doi.org/10.1016/j.meatsci.2014.02.015

Scollan, N. D., Price, E. M., Morgan, S. A., Huws, S. A., \& Shingfield, K. J. (2017). Can we improve the nutritional quality of meat? Proceedingsof the Nutrition Society, 76(4), 603-618. https://doi.org/10.1017/S0029665117001112

Sierra, V., Aldai, N., Castro, P., Osoro, K., Coto-Montes, A., \& Oliván, M. (2008). Prediction of the fatty acid composition of beef by near infrared transmittance spectroscopy. Meat Science, 78(3), 248-255. https://doi.org/10.1016/j. meatsci.2007.06.006 
Snyder, H. (2019). Literature review as a research methodology: An overview and guidelines. Journal of Business Research, 104(July), 333-339. https://doi.org/10.1016/j.jbusres.2019.07.039

Su, H., Sha, K., Zhang, L., Zhang, Q., Xu, Y., Zhang, R., \& Sun, B. (2014). Development of near infrared reflectance spectroscopy to predict chemical composition with a wide range of variability in beef. Meat Science, 98(2), 110-114. https://doi.org/10.1016/j.meatsci.2013.12.019

Su, H., Zhang, S., Li, H., Xie, P., \& Sun, B. (2018). Using near-infrared reflectance spectroscopy to predict physical parameters of beef. Spectroscopy Letters, 51(4), 163-168. https://doi.org/10.1080/00387010.2018.1442355

Tøgersen, G., Isaksson,T., Nilsen, B. N., Bakker, E. A., \& Hildrum, K. I. (1999). On-line NIR analysis of fat, water and protein in industrial scale ground meat batches. Meat Science, 51(1), 97-102. https//doi.org/10.1016/s0309-1740(98)00106-5

Ton, S., De Marchi, M., Manfrin, D., Meneghesso, M., Cassandro, M., \& Penasa, M. (2015). Use of near infrared technology to predict fatty acid groups in commercial ground meat products. Poljoprivreda, 21(Supplement 1), 232-236. https:// doi.org/10.18047/poljo.21.1.sup.55

Troy, D. J., \& Kerry, J. P. (2010). Consumer perception and the role of science in the meat industry. Meat Science, 86(1), 214-226. https://doi.org/10.1016/j.meatsci.2010.05.009

Van-Loo, E. J., Caputo, V., \& Lusk, J. L. (2020). Consumer preferences for farm-raised meat, lab-grown meat, and plant-based meat alternatives: Does information or brand matter? Food Policy, 95, Article 101931. https://doi.org/10.1016/j. foodpol.2020.101931

Warriss, P. D. (2000). Meat science: An introductory text (2 ${ }^{\text {nd }}$ Ed). CABI. https://doi.org/10.1016/S0309-1740(00)00053-X

Weeranantanaphan, J., Downey, G., Allen, P., \& Sun, D. W. (2011). A review of near infrared spectroscopy in muscle food analysis: 2005-2010. Journal of Near Infrared Spectroscopy, 19(2), 61-104. https://doi.org/10.1255/jnirs.924

Willett, W., Rockström, J., Loken, B., Springmann, M., Lang, T., Vermeulen, S., Garnett, T., Tilman, D., DeClerck, F., Wood, A., Jonell, M., Clark, M., Gordon, L. J., Fanzo, J., Hawkes, C., Zurayk, R., Rivera, J. A., De-Vries, W., Sibanda, L. M., ... Murray, C. J. L. (2019). Food in the Anthropocene: the EAT-Lancet Commission on healthy diets from sustainable food systems. The Lancet, 393(10170), 447-492. https://doi.org/10.1016/s0140-6736(18)31788-4

Workman, J. (2016). The concise handbook of analytical spectroscopy : theory, applications, and reference materials. World Scientific Publishing. https://doi.org/10.1142/8800

Yang, S. H., Suhandoko, A. A., \& Chen, D. (2020). Impact of nutritional information on consumers' willingness to pay for meat products in traditional wet markets of taiwan. Foods, 9(8), Article 1086. https://doi.org/10.3390/foods9081086

Young, O., West, J., Hart, A., \& van Otterdijk, F. F. (2004). A method for early determination of meat ultimate pH. Meat Science, 66(2), 493-498. https://doi.org/10.1016/S0309-1740(03)00140-2 